\title{
Learning Vectors (LVs) um Instrumento Automatizado de Avaliação para Suporte a Aprendizagem em EaD *
}

\author{
(Learning Vectors (LVs) an Automatic Evaluation \\ Instrument for Support to EAD Learning *)
}

Gilvandenys Leite Sales - Centro Federal de Educação Tecnológica do Ceará

denyssales@cefetce.br

Giovanni Cordeiro Barroso - Universidade Federal do Ceará gcb@fisica.ufc.br

José Marques Soares - Centro Federal de Educação Tecnológica do Ceará

marques@cefetce.br

Resumo. Este artigo propõe um instrumento de avaliação para ambientes virtuais de aprendizagem, em que o uso de vetores e suas características (intensidade e direção) procuram estabelecer juízo de valor às atividades desenvolvidas em suas ferramentas de interação, tais como: Fóruns, Chats e Portfólio. Pretende-se com este instrumento ampliar as possibilidades de conotação qualitativa a ser dada à avaliação em educação a distância.

Palavras-chave: Educação a distância, Ambientes virtuais de aprendizagem, Instrumento de avaliação, Learning Vectors.

Abstract. This paper proposes an instrument of evaluation for Virtual Learning Environments, where the use of vectors and its characteristics (magnitude and direction) mean to establish judgment of value to the activities developed in the available tools of interaction: Forums, Chats and Portfolio. It is intended with this instrument to extend the possibilities of qualitative connotation to be given to the evaluation in e-learning.

Keywords: e-learning, Virtual learning environments, Learning Vectors.

\section{Introdução}

Face à carência de professores nas mais diversas áreas do saber, medidas vêm sendo tomadas, citam-se a expansão dos cursos presenciais de licenciatura na rede CEFET (Centros Federais de Educação Tecnológica) e a implantação de cursos a distância na modalidade semipresencial da Universidade Aberta do Brasil (UAB).

Esta pesquisa motiva-se por este preocupante quadro de demanda de professores e tem propósitos em colaborar com ferramentas de avaliação que se agreguem aos Ambientes Virtuais de Aprendizagem (AVA). A mesma desenvolve-se como parte de um trabalho de doutorado que se propõe a colaborar com a educação a distância (EaD) através da inclusão nos AVA de instrumentos automatizados de avaliação qualitativa.

\section{Instrumentos de Avaliação em Ambientes Virtuais de Aprendizagem}

Proceedings of the XII SIBGRAPI (October 1999) 101-104 
Ambientes Virtuais de Aprendizagem (AVA) são plataformas de aprendizagem colaborativa dispostas na rede Web para interação de comunidades de aprendizes, que incluem ferramentas para a atuação de um aprendiz autônomo, oferecendo recursos para uma aprendizagem coletiva e/ou individual.

*Este trabalho desenvolve-se como tese de doutorado no Departamento de Engenharia de Teleinformática da Universidade Federal do Ceará.

Com relação à avaliação, comunga-se com as idéias de Zabala (1998) ao expressar que: “(...) a finalidade da avaliação é ser um instrumento educativo que informa e faz uma valoração do processo de aprendizagem seguido pelo aluno, com o objetivo de oportunizar, em todo momento, as propostas educacionais mais adequadas".

Embora os AVA costumem apresentar instrumentos de avaliação, na maioria das vezes eles voltam-se para o caráter quantitativo, limitando-se a informar o número de acessos ou a quantidade de tarefas depositadas no portfólio. Lopes (2007), que apresenta uma revisão bibliográfica acerca de instrumentos de avaliação em AVA, mostra que o paradigma predominante (Quadro 1) ainda é o quantitativo.

\begin{tabular}{|c|c|c|c|c|c|}
\hline Pesquisador & Ferramenta & $\begin{array}{l}\text { Unidade de } \\
\text { avaliação }\end{array}$ & $\begin{array}{l}\text { Paradigma } \\
\text { predominante }\end{array}$ & Perspectiva & Proposta \\
\hline $\begin{array}{l}\text { OTSUKA, } \\
\text { ROCHA }\end{array}$ & $\begin{array}{l}\text { Acessos, } \\
\text { Intermap, } \\
\text { fórum, chat }\end{array}$ & Interações & Quantitativo & Participação & $\begin{array}{l}\text { Multiagentes para análise da } \\
\text { participação }\end{array}$ \\
\hline SILVA & Chat & Mensagem & Quantitativo & Aprendizagem & \begin{tabular}{|l} 
Agente qualificando \\
participante na máquina de \\
estados finitos
\end{tabular} \\
\hline $\begin{array}{l}\text { PIMENTEL, } \\
\text { ESCOVEDO }\end{array}$ & Fórum & Mensagem & Quantitativo & Participação & Notas e comentários \\
\hline SOLLER & Chat & Mensagem & Quantitativo & Colaboração & $\begin{array}{l}\text { Categorização de mensagem e } \\
\text { modelo probabilístico }\end{array}$ \\
\hline GEROSA & Fórum & Mensagem & Quantitativo & Colaboração & Categorização de mensagem \\
\hline $\begin{array}{l}\text { CAVAROLI \& } \\
\text { COELLO }\end{array}$ & Fórum & Mensagem & Quantitativo & Participação & $\begin{array}{l}\text { Categorização de mensagens } \\
\text { e alertas a partir da avaliação } \\
\text { dos pares }\end{array}$ \\
\hline $\begin{array}{l}\text { HENRI, } \\
\text { MASON, } \\
\text { WEBB, } \\
\text { NEWMAN \& } \\
\text { COCHRANE }\end{array}$ & Fórum & Mensagem & Qualitativo & $\begin{array}{l}\text { Análise do } \\
\text { significado / } \\
\text { Aprendizagem }\end{array}$ & Categorização de mensagens \\
\hline STAHL & Fórum & Mensagem & Qualitativo & $\begin{array}{l}\text { Análise do } \\
\text { significado / } \\
\text { Colaboração }\end{array}$ & Rede de referência semântica \\
\hline $\mathrm{CORICH}$ & Fórum & Mensagem & Qualitativo & $\begin{array}{l}\text { Análise do } \\
\text { significado / } \\
\text { Aprendizagem }\end{array}$ & CMC e Community of Learning \\
\hline
\end{tabular}

Quadro 1 - Propostas e ferramentas de avaliação

O Quadro 1 acima inclui pesquisadores de importantes AVA atualmente em ação e com uma vasta ficha de registros de importantes contribuições ao longo de anos, citam-se o AulaNet / PUC-Rio (Gerosa et al., 2004; Pimentel et al., 2004; Escovedo et al., 2006), TelEduc / UNICAMP (Otsuka e Rocha, 2005; Rocha, 2006) e WebCT / PUC-Campinas (Cavaroli e Coelho, 2004).

Portanto, ainda não se contemplou efetivamente uma avaliação qualitativa e formativa em $\mathrm{EaD}$ que, além de mediar a aprendizagem, também reduza o trabalho offline do professor-tutor.

\section{Learning Vectors (Vetores Aprendizagem)}


Os Learning Vectors (LVs) são representações geométricas vetoriais concebidos para auxiliar o processo de avaliação em Ambientes Virtuais de Aprendizagem (AVA), cuja implementação, testes e refinamentos estão sendo feitos no ambiente MOODLE do Centro Federal de Educação Tecnológica do Ceará (CEFETCE).

Como instrumento de avaliação online os LVs reúnem aspectos qualitativos e quantitativos no acompanhamento de desempenho do aluno/usuário. Eles podem possibilitar feedbacks constantes das ações do aprendiz no ambiente virtual de aprendizagem, além de reduzir a sobrecarga gerada para o professor-tutor em cursos a distância.

A concepção epistemológica dos LVs busca referência em teóricos interacionistas, cuja construção do conhecimento ocorre na interação Sujeito X Objeto do conhecimento (Piaget, 1973), na relação sociocultural Sujeito X Sujeito (Vygotsky, 1998), na contemporaneidade Sujeito X Coletividade (Lèvy, 2004) e na diversidade Sujeito X Complexidade (Morin, 1991), afinal lidar com a aprendizagem em sala de aula, seja presencial ou virtual, constitui um sistema dinâmico imprevisível muitas vezes cheio de incertezas.

Como forma de exemplificar esta proposta de LVs (Figura 1), introduzem-se vetores que representem as ações do aprendiz em sua interação com fóruns $(\mathrm{F})$, portfólios $(\mathrm{P})$ e chats $(\mathrm{C})$, bem como vetores informativos do número total de faltas (NTF) e da nota da avaliação presencial (AP). Inicialmente os $L V s \mathrm{~F}, \mathrm{P}, \mathrm{C}$ e NTF formam $90^{\circ} \mathrm{com}$ a horizontal, e o vetor AP, por seu maior significado (Brasil, 2006) em cursos semi-presenciais, forma $0^{\circ}$ com a horizontal.

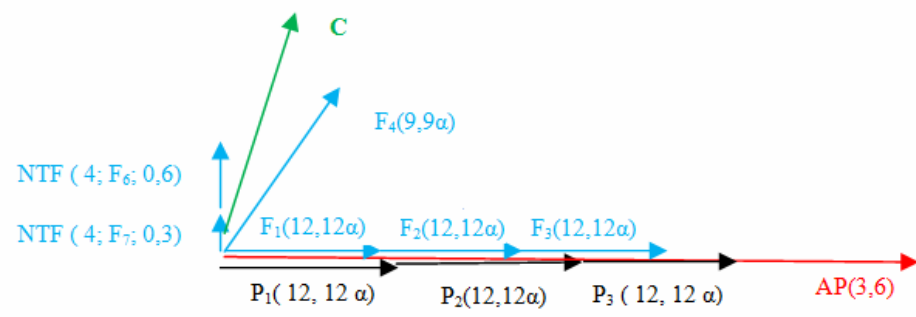

Figura 1 - Learning Vectors (LVs)

O ângulo que os LVs (F, P e C) formam com a vertical variará em função do desempenho dos alunos/cursistas durante as atividades. Seu valor será múltiplo do ângulo padrão $\alpha$ definido empiricamente como $7,5^{\circ}$ a partir da observação de experiências práticas de tutores da Universidade Aberta do Brasil (UAB). Enquanto que os LVs NTF e AP permanecem em direções fixas.

As inserções do aluno e participação no curso, avaliadas continuamente pelo professor-tutor, serão categorizadas segundo uma escala Likert de 5 pontos, associada a uma escala icônica (Figura 2), do tipo: 


\begin{tabular}{|l|l|l|}
\hline & & $\begin{array}{l}\text { saudações, recados e/ou agradecimentos com interações/postagens que em nada } \\
\text { contribuem para a discussão/aprendizagem do tema proposto. }\end{array}$ \\
\hline$(2)$ & $\begin{array}{l}\text { Fraco } \\
\text { Com reflexões vazias de conteúdo suas interações/postagens pouco agregam valor à } \\
\text { discussão/aprendizagem do tema proposto. }\end{array}$ \\
\hline$(3)$ & $\begin{array}{l}\text { Regular } \\
\text { A partir de razoáveis reflexões suas interações/postagens acerca do tema proposto } \\
\text { para a discussão/aprendizagem são limitadas e de forma superficial. }\end{array}$ \\
\hline (5) & $\begin{array}{l}\text { Bom } \\
\text { A partir de boas reflexões suas interações/postagens apresentam respostas e } \\
\text { comentários significativos para discussão/aprendizagem do tema proposto. }\end{array}$ \\
\hline $\begin{array}{l}\text { Muito Bom } \\
\text { A partir de reflexões mais aprofundadas suas interações/postagens podem apresentar, } \\
\text { além de respostas e comentários, significativos questionamentos ou mesmo sínteses de } \\
\text { idéias que surgem na discussão/aprendizagem do tema proposto gerando mais } \\
\text { interações. }\end{array}$ \\
\hline
\end{tabular}

Figura 2 - Escala Likert-Icônica

Esta escala surgirá, conforme Figura 3, sempre que o aluno/cursista fizer alguma inserção, seja nos fóruns, chats ou portfólio. A intenção é transformar a avaliação em processo formativo.

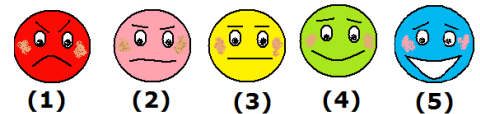

Figura 3 - Apresentação da Escala Likert-Icônica

O ângulo dos LVs Fórum e Chat gira em sentido horário de um múltiplo do ângulo $\alpha$ para as categorias (3), (4) e (5) da escala Likert-Icônica e no sentido antihorário de uma fração do ângulo $\alpha$ para avaliações correspondentes aos dois pontos inferiores da escala (1) e (2).

Cada vetor F, P, C e AP terá um fator multiplicador determinado pela significância da atividade. Significância $\mathbf{S}$ é um escalar relacionado à quantificação da atividade definida inicialmente pelos gestores do curso ou disciplina.

O vetor resultante $\mathbf{L V} \mathbf{Q}$ (Quociente de Aprendizagem) deve ser normalizado numa escala de 0 a 10 e representa a nota final do aluno. O módulo do vetor $\mathbf{Q}$, ou seja, seu valor numérico, está relacionado ao quantitativo/qualitativo de aprendizagem.

\section{1 - O Vetor LV Fórum (LV F)}

Denomina-se $\mathrm{P}_{0}$ à primeira Interação Principal(IP) postada no Fórum, ou seja, aquela que revela a compreensão do aluno/interagente acerca do tema em discussão. Sua categorização associada à escala Likert - Icônica é mostrada na Figura 4.

\begin{tabular}{|l|l|l|l|}
\hline & Categorias & \multicolumn{2}{|c|}{$\mathrm{P}_{0} /$ Ângulo } \\
\hline (1) & 00 & $0 \alpha$ & $0-0^{\circ}$ \\
\hline
\end{tabular}




\begin{tabular}{|l|l|l|l|}
\hline$(2)$ & 0 & $2 \alpha$ & $2-15^{\circ}$ \\
\hline$(3)$ & 60 & $4 \alpha$ & $4-30^{\circ}$ \\
\hline$(4)$ & $6 \alpha$ & $6-45^{\circ}$ \\
\hline$(5)$ & 0 & $8 \alpha$ & $8-60^{\circ}$ \\
\hline
\end{tabular}

Figura 4 - Primeira Interação Principal no Fórum

Um aluno que faz uma primeira IP de boa qualidade no fórum, categoria (5) da Figura 2, em que, além de se posicionar acerca do tema proposto para discussão, comenta, questiona e/ou sintetiza as inserções de seus colegas tem seu LV F incrementado de 8 pontos no seu módulo e direção de $60^{\circ}$ com a vertical (Ver Figura 4).

Denomina-se $\mathrm{N}_{0}$ à primeira Interação Secundária(IS) postada no Fórum, ou seja, aquela que vem como resposta, comentário, sugestão, crítica, concordância, etc. a alguma interação principal já depositada no Fórum, com fins de provocar dinamismo nas discussões e gerar mais interações acerca do tema em estudo. Sua categorização associada à escala Likert - Icônica é mostrada na Figura 5.

\begin{tabular}{|c|c|c|c|}
\hline \multicolumn{2}{|c|}{ Categorias } & \multicolumn{2}{|c|}{$\mathrm{N}_{0} /$ Ângulo } \\
\hline$(1)$ & 00 & $0 \alpha$ & $0-0^{\circ}$ \\
\hline$(2)$ & 00 & $1 \alpha$ & $1-7,5^{\circ}$ \\
\hline$(3)$ & 0 & $2 \alpha$ & $2-15^{\circ}$ \\
\hline$(4)$ & 0 & $3 \alpha$ & $3-22,5^{\circ}$ \\
\hline$(5)$ & 0 & $4 \alpha$ & $4-30^{\circ}$ \\
\hline
\end{tabular}

Figura 5 - Primeira Interação Secundária no Fórum

Um aluno que faz uma primeira IS limitando-se apenas a concordar com idéias postadas por outro interagente sem agregar valor ao tema proposto, categoria (1) da Figura 2, não tem incremento de módulo nem mudança na direção de seu LV F (Ver Figura 5). 
É importante observar que a primeira interação principal $\left(\mathrm{P}_{0}\right)$ (Figura 4$)$ tem, correspondentemente a cada categoria, peso maior comparado à interação secundária $\left(\mathrm{N}_{0}\right)$ (Figura 5).

Note que o valor $12 \alpha$ corresponde a um ângulo de $90^{\circ}$. Se, após o aluno postar suas primeiras interações primária $\left(\mathrm{P}_{0}\right)$ e secundária $\left(\mathrm{N}_{0}\right)$ é atingido o limite de $12 \alpha$, o LV Fórum registra o valor máximo para aquela atividade e cessa seu incremento, visto que a direção do mesmo será a horizontal.

Caso o aluno não tenha atingido o limite de $12 \alpha$, após suas primeiras interações $\left(\mathrm{P}_{0}\right.$ e $\mathrm{N}_{0}$ ), para cada nova interação qualificada com categoria (3), (4) ou (5), o vetor incrementa de $1 \alpha$ (o vetor LV Fórum gira de $\alpha$ e seu módulo cresce de uma unidade) até que se complete o limite supracitado. Mas para cada interação categorizada com categoria (1) ou (2), o vetor LV Fórum gira no sentido anti-horário de um ângulo $\alpha / 2$ sem modificar seu módulo, até o limite de $90^{\circ}$.

Prevê-se que esta medida incite a interatividade, uma vez que, não se atingindo o limite de $12 \alpha$, o aluno procurará fazer mais inserções qualificadas nos fóruns no intuito de atingir uma maior pontuação. Por exemplo, um aluno que tenha obtido na $\mathrm{P}_{0}$ a categoria (4) e na $\mathrm{N}_{0}$ a categoria (3), ou seja, $6 \alpha+2 \alpha=8 \alpha$, precisa fazer, no mínimo, mais 4 interações no fórum em curso categorizadas como (3), (4) ou (5) para atingir a pontuação máxima.

Os números $\mathrm{P}_{0}$ e $\mathrm{N}_{0}$, respectivamente relacionados à interação primária (IP) ou interação secundária (IS), que precedem os ângulos $\alpha$ nas Figuras 4 e 5, juntamente com os acréscimos unitários de $1 \alpha$, compõem o módulo do vetor LV Fórum.

Devidamente normalizado numa escala de 0 a 10 a nota de cada Fórum será representada pelo módulo do componente horizontal do LV Fórum.

\subsection{O Vetor LV Portfólio}

O Portfólio, assim como o Fórum, por se constituir como ferramenta assíncrona, disponibiliza ao aluno mais tempo para sua postagem, entretanto não possui a mesma interatividade, razão pela qual o limite de $12 \alpha$ pode ser atingido na primeira postagem e levar o LV Portfólio a cessar sua incrementação.

A postagem no portfólio obedece aos seguintes critérios para pontuação (Figura 6):

\begin{tabular}{|l|l|l|l|}
\hline \multicolumn{2}{|l|}{ Categorias } & \multicolumn{2}{|l|}{$\mathrm{PP} /$ Ângulo } \\
\hline$(1)$ & 00 & $0 \alpha$ & $0-0^{\circ}$ \\
\hline$(2)$ & 0 & $3 \alpha$ & $\begin{array}{l}3- \\
22,5^{\circ}\end{array}$ \\
\hline$(3)$ & 00 & $6 \alpha$ & $6-45^{\circ}$ \\
\hline$(4)$ & 0 & $9 \alpha$ & $\begin{array}{l}9- \\
67,5^{\circ}\end{array}$ \\
\hline
\end{tabular}




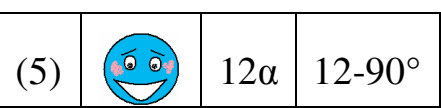

Figura 6 - Categorização para Postagem Primária no Portfólio

Como esta proposta de avaliação é formativa e mediadora da aprendizagem, o limite máximo de $12 \alpha$, mesmo que não tenha sido atingido, pode ainda ser atingido, desde que se possibilite o refazer por meio de uma segunda e última postagem.

A segunda postagem de uma mesma atividade no portfólio é optativa para o aluno/interagente obedecendo aos seguintes critérios de pontuação complementar (Figura 7):

\begin{tabular}{|l|l|l|l|}
\hline \multicolumn{2}{|c|}{ Categorias } & \multicolumn{2}{l|}{ PS/Ângulo } \\
\hline$(1)$ & 0 & $0 \alpha$ & $0-0^{\circ}$ \\
\hline$(2)$ & 0 & 0 & $1-7,5^{\circ}$ \\
\hline$(3)$ & 0 & $2 \alpha$ & $2-15^{\circ}$ \\
\hline$(4)$ & 0 & $3 \alpha$ & $3-22,5^{\circ}$ \\
\hline$(5)$ & 0 & $4 \alpha$ & $4-30^{\circ}$ \\
\hline
\end{tabular}

Figura 7 - Categorização para Postagem Secundária no Porttólio

Os números PP e PS, respectivamente relacionados à postagem primária e postagem secundária, que precedem os ângulos $\alpha$ nas tabelas das Figuras 6 e 7 compõem o módulo do vetor LV Portfólio.

A nota de cada Portfólio numa escala de 0 a 10 será representada pelo módulo do componente horizontal do LV Portfólio.

\section{4 - O Vetor LV Chat}

O Chat por se constituir como ferramenta síncrona, cuja dinâmica é muito acelerada, deve ser visível apenas para o professor-tutor durante sua realização. Ao final de uma sessão de Chat o corresponde LV pode surgir para o aluno/interagente, uma vez que o professor-tutor o tenha pontuado durante o desenrolar de sua realização.

Caso o Chat não venha a computar nota ele pontua apenas como indicador de freqüência do aluno-interagente. 8):

As interações nos chats obedecem aos seguintes critérios para pontuação (Figura

\begin{tabular}{|c|c|c|c|}
\hline \multicolumn{2}{|c|}{ Categorias } & \multicolumn{2}{|c|}{$\mathrm{C}_{\mathrm{i}} /$ Ângulo } \\
\hline$(1)$ & $0 \alpha$ & $0-0^{\circ}$ \\
\hline
\end{tabular}




\begin{tabular}{|c|c|c|c|}
\hline (2) & 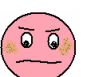 & $0,5 \alpha$ & $0,5-3,75^{\circ}$ \\
\hline (3) & (ब) & $1 \alpha$ & $1-7,5^{\circ}$ \\
\hline (4) & (6) & $1,5 \alpha$ & $1,5-11,25^{\circ}$ \\
\hline (5) & 60 & $2 \alpha$ & $2-15^{\circ}$ \\
\hline
\end{tabular}

Figura 8 - Categorização para Chats

O limite máximo de $12 \alpha$ pode ser atingido com 6 interações do tipo (5) ou 8 interações do tipo (4), ou qualquer combinação delas com as demais categorias. Quando isto ocorre o vetor LV Chat registra o valor máximo para aquela sessão de chat e cessa sua incrementação.

Para cada interação categorizada como (1) o vetor LV Chat gira no sentido antihorário de um ângulo $\alpha / 4$ sem alterar seu módulo.

Os números $\mathrm{C}_{\mathrm{i}}$ que precedem os ângulos $\alpha$ na tabela da Figura 8 compõem o módulo do vetor LV Chat.

A nota de cada Chat - NC numa escala de 0 a 10 será representada pelo módulo do componente horizontal do LV Chat.

\section{O Indicador qualitativo não-linear de aprendizagem - Fator $\beta$}

Fundamentado no princípio de que comunidades de aprendizagem virtuais são sistemas dinâmicos complexos e que a aprendizagem não é um processo linear, portanto exige um indicador não-linear de avaliação (Araújo, 2004; Araújo e Filho, 2005), bem como na dimensão Positividade/Negatividade (P/N) do modelo de avaliação de desempenho denominado Meta Learning (Fredrickson e Losada, 2004; Losada 1999) proposto para avaliação de equipes. Desenvolveu-se um indicador qualitativo não-linear de aprendizagem do aluno/cursista intitulado Fator $\beta$.

Devidamente adaptado aos LVs (Figura 9) tal fator relaciona os resultados positivos ou negativos das contribuições dos alunos nas atividades colaborativas desenvolvidas.

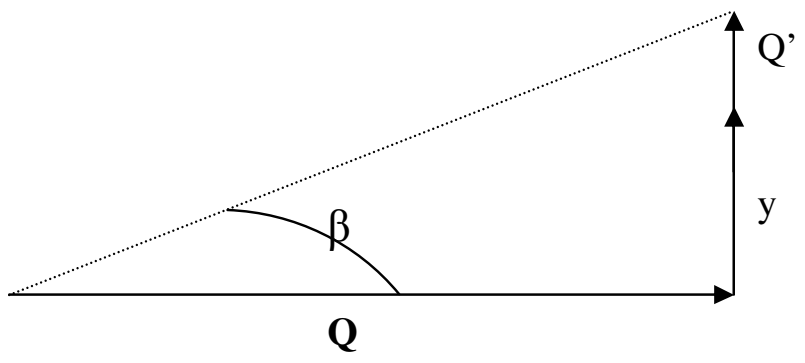

Figura 9 - Fator $\beta$ 
Toma-se como contribuição positiva ou Positividade (P) o vetor LV Q, que é formado pela projeção horizontal dos LVs Fóruns, Portfólios e Chats acrescido da avaliação presencial (AP), quanto mais estes vetores se projetam horizontalmente maior a qualidade das interações. E como contribuição negativa ou Negatividade (N) a projeção vertical dos LVs Fóruns, Portfólios e Chats (Q') somados aos pontos perdidos por ausência em alguma atividade (y). O Fator $\beta$, classificado abaixo (Figura 10), exprime que, quanto menor for seu valor maior será qualitativamente o desempenho do aluno/cursista. Estes valores foram resultados de uma aplicação dos LVs em duas turmas semi-presenciais da UAB.

\begin{tabular}{|c|c|c|}
\hline \multicolumn{2}{|c|}{ Categorias } & Fator $\beta$ \\
\hline (1) & & $\beta \square 15^{\circ}$ \\
\hline (2) & $(\bar{\theta})$ & $7^{\circ}<\beta \leq 15^{\circ}$ \\
\hline (3) & $\bar{\theta} \bar{\theta}$ & $4^{\circ}<\beta \leq 7^{\circ}$ \\
\hline (4) & (6) & $2^{\circ}<\beta \leq 4^{\circ}$ \\
\hline (5) & (6) & $0^{\circ} \leq \beta \leq 2^{\circ}$ \\
\hline
\end{tabular}

Figura 10 - Classificação do ângulo $\beta$

Após a correção da avaliação presencial e lançamento de sua nota no sistema, um agente inteligente coleta os dados, aluno por aluno, e apresenta finalmente ao lado da representação gráfica abaixo, que contém, além da representação iconográfica associada ao Fator $\beta$ (Figura 11), a tripla $\left(\mathrm{N}^{\circ}\right.$ Total de Faltas; Atividades ausentes; pontos perdidos) e o Indicador de Negatividade nas Interações (Q') na vertical e na horizontal a dupla (Quociente de Aprendizagem - Q; Conceito). No caso do aluno não alcançar a média para aprovação ele fará jus à Avaliação Presencial Final $\left(\mathrm{AP}_{\mathrm{F}}\right)$ e sua nova média após estes estudos de recuperação comporá a dupla (Quociente de Aprendizagem após Recuperação - $\mathrm{Q}_{\mathrm{R}}$; Conceito). O Quociente de Aprendizagem Q ou $\mathrm{Q}_{\mathrm{R}}$ deve ser apresentado como uma soma das contribuições nas Atividades Colaborativas (AC) de Fóruns, Chats e Portfólios mais a Avaliação Presencial, ou seja, $\mathrm{Q}=\mathrm{AC}+\mathrm{AP}$.

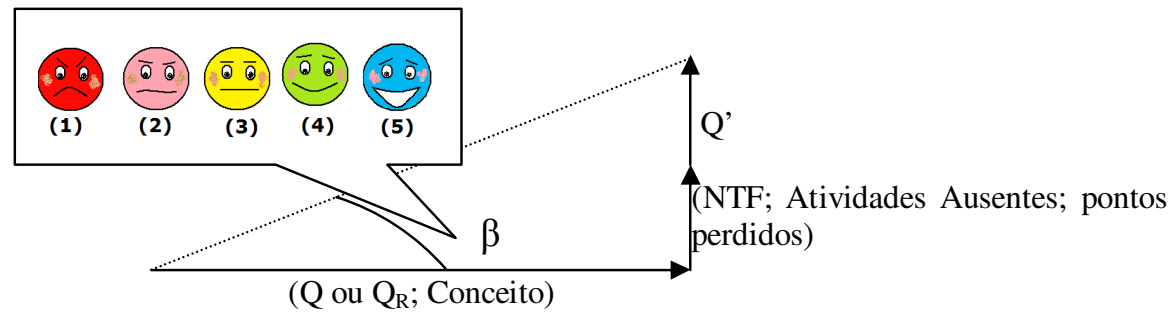

Figura 11 - Apresentação Final do Desempenho do Aluno

Sugere-se que o conceito atribuído ao aluno siga a seguinte classificação: 
A - Aluno aprovado por média - Q $\geq 7,0$;

- B - Aluno aprovado após recuperação final - $\mathrm{Q}_{\mathrm{R}} \geq 5,0$;

- $\mathrm{R}$ - Aluno reprovado por nota $-\mathrm{Q}_{\mathrm{R}} \leq 4,9$;

- F-Aluno reprovado por faltas - NTF $\square 25 \%$ da Carga Horária Total.

Como exemplo apresenta-se o resultado final de um aluno (Figura 12), devidamente tutoriado em um curso da UAB, correspondente aos LVs da Figura 1, anteriormente apresentada e vislumbra-se a contribuição que este instrumento automatizado de avaliação pode trazer a EaD.

Observa-se que os LVs constituem um dossiê do aluno cursista interpretável para qualquer um que o acesse. Quantitativamente sabe-se que ele tirou 5,94 na avaliação presencial, 2,34 nas atividades colaborativas a distância. Qualitativamente suas interações geraram um fator negativo de 0,27 , com um fator beta de $11,1^{\circ}$ que o classifica como aluno fraco. Vê-se ainda que ele não participou nos fóruns de números 6 e 7, o que lhe acarretou 0,9 pontos perdidos e um total de 8 faltas. Este quadro conduz ao conceito $\mathrm{R}$ - reprovado.

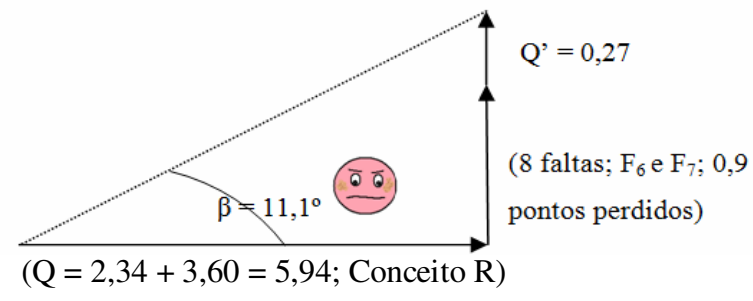

Figura 12 - Apresentação do desempenho final

\section{Considerações Finais}

Este trabalho de pesquisa aponta caminhos para se construir instrumentos de avaliação que venham a se agregar aos Ambientes Virtuais de Aprendizagem, e revelem não só o caráter quantitativo, como também o qualitativo do processo de avaliação. Isto poderá trazer mais credibilidade aos que buscam esta modalidade de ensino.

A proposta dos Learning Vectors teve suas primeiras aplicações nos cursos semipresenciais oferecidos pela Universidade Aberta do Brasil e, até agora, tem se mostrado um instrumento de grande valia para os ambientes virtuais, servindo não somente ao aluno, que tem um acompanhamento contínuo de suas atividades, bem como aos tutores por viabilizar o processo de avaliação. Entretanto, mais testes de aplicação devem ser conduzidos para validação deste instrumento de avaliação.

A análise dos resultados das primeiras aplicações dos LVs revelam não somente sua dimensão qualitativa, mas também seu caráter de sociabilidade e transparência ao permitir a quem os acesse, portanto deixa de ser exclusividade do professor-tutor, fazer julgamentos e inferências acerca do desempenho dos alunos ao final de uma atividade. 
Espera-se reduzir a sobrecarga off-line de trabalho dos professores-tutores no acompanhamento das participações dos alunos/interagentes nas atividades virtuais colaborativas e contribuir para a formação online, principalmente de licenciados nas mais diversas áreas do saber, atribuindo aos cursos semipresenciais da UAB mais dinamismo e credibilidade.

Por fim, o uso de ferramentas telemáticas na educação a distância não prescinde da mediação humana. Esta sinergia pode ser a propulsora de inovações pedagógicas a serviço da construção de saberes e de uma formação autônoma.

\section{Referências}

Araújo, L. H. L. Uma aplicação da dinâmica não-linear para avaliação de desempenho de comunidades virtuais de aprendizagem. Além da tela do computador: linguagem, emocionalidade e corporalidade. Orientador: Gentil José de Lucena Filho. Brasília: UCB, 2004, 194p. Dissertação. (Mestrado em Gestão do Conhecimento e Tecnologia da Informação). Disponível em:< http://bdtd.ucb.br/tede/tde_busca/arquivo.php?codArquivo=185>. Acesso em: 26 maio 2008.

Araújo, L. H. L. e Filho, G. J. L. Comunidades virtuais de aprendizagem: novas dinâmicas de aprendizagem exigem novas formas de avaliação. In: Simpósio Brasileiro sobre Informática na Escola. 2005.

Brasil 2006. Decreto No 5622 de 19/12/2005. Disponível em: <http://portal.mec.gov.br >. Acesso em: 20/06/2007.

Cavaroli, J. T. e Coello, J. M. A. Alerts fórum: um sistema emissor de alertas a partir da avaliação de mensagens categorizadas em fóruns de discussão. In: CONGRESSO IBEROAMERICANO DE INFORMÁTICA EDUCATIVA, 7, 2004, Monterrey. Actas... Monterrey, 2004. p. 39-47.

Escovedo, Tatiana et al. . Avaliei, avaliei, ensinei? Investigações sobre avaliação nas conferências de um curso online. In: WORKSHOP DE INFORMÁTICA NA EDUCAÇÃO. Anais... Campo Grande, MS: SBC, 2006. p. 94-101. WIE 2006

Gerosa, M. A., Fuks, H. e Lucena, C. J. P. Estruturação e categorização de mensagens em ferramentas de comunicação textuais assíncronas. In: WORLD CONGRESS ON ENGINEERING AND TECHNOLOGY EDUCATION - WCETE'2004, Santos Proceedings... Santos: COPEC/IEEE, 2004.

Lopes, M. S. S. Avaliação da aprendizagem em atividades colaborativas em EaD viabilizada por um fórum categorizado. Rio de Janeiro, 2007. 168 f.; Dissertação. Universidade Federal do Rio de Janeiro, Instituto de Matemática, Núcleo de Computação Eletrônica.

Losada, M. The complex dynamics of high performance teams. Mathematical and Computer Modelling, 30, 1999, p.179-192.

Fredrickson, B. L. e Losada, M. Positive Affect and the Complex Dynamics of Human

Flourishing. American Psychologist, vol. 60, no. 7, 2005, pp. 678-686.

Lèvy, P. As tecnologias da inteligência. Rio de Janeiro: Editora 34. 2004.

V. 6 № 1, Julho, 2008 
Morin, E. Introdução ao Pensamento Complexo. Lisboa, Instituto Piaget. 1991.

Piaget, J. Problemas de Psicologia Genética. São Paulo, Florense. 1973.

Pimentel, M.G., Fuks, H. e Lucena, C.J.P. Avaliação da Participação em Conferências Textuais Assíncronas, WORKSHOP DE INFORMÁTICA NA ESCOLA, Salvador. Anais... Salvador: SBC, 2004. p. 112.

Otsuka, J. L. e Rocha, H. V. Um modelo de suporte à avaliação formativa para ambientes de EaD. Campinas: Instituto de Computação-Unicamp. (Relatório Técnico IC-05-11). 2005.

Rocha, V. et al. Avaliação online: o modelo de suporte tecnológico do projeto Teleduc. In: SILVA, Marco; SANTOS, Edméa (Org.). Avaliação da aprendizagem em educação online. São Paulo: Loyola. 2006, p. 347-368.

Vygotsky, L. S. A Formação Social da Mente. São Paulo, Martins Fontes. 1998.

Zabala, A. A Prática Educativa: como ensinar. Trad. Ernani F. da F. Rosa. Porto Alegre: ArtMed. 1998. 